\title{
Carrier and Photon Analyses of Photonic Microlasers by Two-Dimensional Rate Equations
}

\author{
Kengo Nozaki and Toshihiko Baba, Member, IEEE
}

\begin{abstract}
In photonic crystal (PC) microlasers with point defect cavities, effective carriers are reduced by the leakage to outside of the defect, surface recombination, spatial carrier hole burning, and Auger recombination. To estimate these effects, we calculated carrier and photon behavior by solving two-dimensional rate equations in space and time domains. The result clearly shows these effects and their dependence on cavity structure, pump area, and so on. Compared with that for the microdisk laser, higher threshold values are estimated for PC microlasers. However, a comparably low threshold density and a high efficiency are expected for the quasi-periodic PC microlaser, because the hole burning by the whispering gallery mode of this cavity suppresses the carrier leakage.
\end{abstract}

Index Terms-Carrier diffusion, finite-difference time-domain (FDTD), microdisk, microlaser, photonic crystal (PC), quasiperiodic photonic crystal (QPC), rate equation, surface recombination velocity, whispering gallery mode.

\section{INTRODUCTION}

$\mathbf{S}$ EMICONDUCTOR photonic microlasers are expected to realize the low threshold lasing due to their small cavities and the high-efficiency and high-speed operation due to the enhanced spontaneous emission rate by the Purcell effect [1]-[4]. Compared with microdisk lasers (MDLs) surrounded by air [4]-[7] and vertical-cavity surface-emitting lasers (VCSELs) with one-dimensional Bragg mirrors [8], [9], photonic crystal (PC) lasers [10]-[12] with point defect cavities are more advantageous for the ultimate reduction in cavity size by using the photonic bandgap (PBG). Because of this reason, a very low threshold [13] and the strong Purcell effect [14] have been demonstrated, and a cavity quantum electrodynamic effect (e.g., strong coupling of the cavity with a quantum dot) has been expected [15]-[17]. On the other hand, quasi-periodic photonic crystals (QPCs) [18]-[20], which have high order rotational and line symmetries, are other candidates for the ultrasmall cavity. Recently, we have demonstrated the lasing action in a QPC microlaser [20]. However, most of these devices are composed of a III-V semiconductor slab, a hole array, and a hole-missing defect cavity. Therefore, part of carriers inside the defect cavity leak out by the diffusion and are lost by the

Manuscript received July 2, 2004; revised December 15, 2004. This work was supported in part by the IT Program and The 21st Century COE Program, both from the Ministry of Education, Culture, Sports, Science and Technology, in part by the Japan Society for the Promotion of Science under Grant-In-Aid 16206008 , and in part by the CREST Project of Japan Science and Technology Corporation.

The authors are with the Yokohama National University, Department of Electrical and Computer Engineering, Yokohama 240-8501, Japan (e-mail: d05sd106@ynu.ac.jp; baba@ynu.ac.jp).

Digital Object Identifier 10.1109/JSAC.2005.851162 surface recombination at sidewalls of holes. This process results in a higher threshold and lower efficiency. The estimation of such useless carriers is an important issue, but has never been discussed yet. In general, rate equations for carriers and photons are used for analyzing lasing characteristics [2], [21], [22]. However, the simple analysis cannot precisely model complex structures of PCs and QPCs. In this work, we performed the finite-difference rate equation analysis with two-dimensional (2-D) cavity models and laser mode profiles. The behavior of carriers and photons including carrier diffusion, surface recombination, spatial hole burning, and Auger recombination were investigated in detail, and the threshold and internal efficiency in these microlasers were estimated. As the first step of this kind of analysis, we employed four sample models of microcavities with a fixed cavity $Q$ factor and spontaneous emission factor. This means that we neglected the unique behavior of spontaneous emission such as the thresholdless lasing and the Purcell effect. We also ignored the thermal heating, which is important for estimating the continuous wave lasing condition but requires a large computational task for getting self-consistent results.

In the next section, we first explain the fundamental formulas and parameters used in the analysis. Then, in Section III, we present some calculated results of carrier distributions and lasing characteristics. In Section IV, we show the dependence on surface recombination velocity, carrier diffusion constant, and cavity $Q$ factor. Finally, in Section V, we discuss the internal quantum efficiency and the effective surface recombination velocity including the carrier leakage effect, and summarize the advantages and disadvantages of each structure.

\section{FORMULAS AND PARAMETERS}

Rate equations for the carrier density $N(x, y ; t)$ and the photon density $S(t)$ (number of photons divided by the volume of active region) with respect to positions $x$ and $y$ and time $t$ are expressed as [21], [22]

$$
\begin{aligned}
\frac{d N}{d t}= & \frac{P}{\hbar \omega}-\Gamma_{z} G S|E|^{2} A_{m}-\frac{N}{\tau_{c}}+D \nabla^{2} N \\
\frac{d S}{d t}= & \int_{\text {Act. }} \Gamma_{z} G S|E|^{2} d x d y \\
& +\frac{C}{\Gamma_{x y}} \int_{\text {Act. }} \frac{N|E|^{2}}{\tau_{\mathrm{sp}}} d x d y-\frac{S}{\tau_{\mathrm{ph}}}
\end{aligned}
$$

where $P(x, y ; t)$ is the pump power density, $\hbar \omega$ the pump energy, $G(N)$ the gain coefficient, $A_{m}$ the effective modal area, $\tau_{c}(x, y ; t)$ the carrier lifetime, $\tau_{\mathrm{sp}}(x, y ; t)$ the spontaneous emission lifetime, $\tau_{\mathrm{ph}}$ the photon lifetime, $D$ the carrier diffusion 
constant, and $C$ the spontaneous emission factor. $E(x, y ; t)$ is the electric field distribution of the laser mode, which is normalized as

$$
\iint_{\text {Cavity }}|E|^{2} d x d y=1
$$

where $E$ is determined by the cavity structure, namely, the refractive index distribution. More accurately, $E$ is slightly changed by the gain, absorption, and carrier-induced index change, which are dependent on $N$. However, in cavities discussed in this paper, the mode is strongly influenced and almost determined by the very large index difference between the semiconductor and air. Therefore, we can consider $E$ to be independent of time $t$ in the rate equations. $\Gamma_{z}$ and $\Gamma_{x y}$ are confinement factors of $E^{2}$ into the active region. Even though the infinite uniformity is assumed in the perpendicular direction ( $z$ direction) in the 2-D analysis, the penetration of $E^{2}$ from the semiconductor slab to air in actual devices reduces the modal gain. $\Gamma_{z}$ is used to express this reduction. The boundary condition at the edge of holes is expressed as

$$
e_{0} N v_{s}=-e_{0} D \nabla N
$$

where $e_{0}$ is the electron charge and $v_{s}$ is the surface recombination velocity.

In the computation procedure, cavity models are assigned in the 2-D space divided by the small unit cell. The position $(x, y)$ is expressed by the discrete coordinate $(i, j)$ and the cell size $\Delta x$ and $\Delta y$ as $(i \Delta x, j \Delta y)$, respectively. The time $t$ is expressed by the step number $n$ and the unit time $\Delta t$ as $n \Delta t . N$ and $S$ are denoted as $N_{i, j}^{n}$ and $S^{n}$, respectively, and derivatives in (1) and (2) are expressed in finite different formulas. Since $E$ is independent of $N$ and $S, E_{i, j}$ is calculated by the so-called finite-difference time-domain (FDTD) method before the rate equation analysis. Thus, (1) and (2) are solved against the pumping condition expressed by $P_{i, j}^{n}$, and dynamic behavior of $N_{i, j}^{n}$ and $S^{n}$ after any time steps are obtained. The lasing threshold is estimated from the photon density at the steady state.

In overall calculations in this paper, we modeled the GaInAsP slab with compressively strained quantum wells (CS-QWs) [21]. In the FDTD analysis, a standard equivalent modal index of the slab of 2.73 was used for the background medium, and those of holes were set to 1.0. In the rate equation analysis, uniform circular photopumping at a wavelength of $0.98 \mu \mathrm{m}$ with a diameter of $2 R_{e x}$ was considered. $\Gamma_{z}$ was set to 0.079 as a typical value for the slab with CS-QWs. On the other hand, $\Gamma_{x y}$ was directly obtained by the FDTD analysis. $A_{m}$ is given by $4 / E_{\max }$, where $E_{\max }$ is the maximum electric field determined by (3). The gain $G(N)$ was approximated by the logarithmic function $G_{0}\left(c / n_{g}\right) \ln \left(N / N_{0}\right)$, where $c$ is the light velocity in vacuum, $n_{g}$ the group index, and $N_{0}$ the transparent carrier density. For the CS-QWs, we set $G_{0}=1500 \mathrm{~cm}^{-1}, n_{g}=3.5$, and $N_{0}=1.5 \times 10^{18} \mathrm{~cm}^{-3}$. The carrier lifetime $\tau_{c}$ has relation $\tau_{c}^{-1}=\tau_{n r}^{-1}+\tau_{\mathrm{sp}}^{-1}$ with the nonradiative recombination lifetime $\tau_{n r}$ and the spontaneous emission lifetime $\tau_{\mathrm{sp}}$. Since the surface recombination is taken into account in (4), $\tau_{n r}^{-1}$ only includes the Auger recombination $C_{A} N^{2}$, where the Auger coefficient $C_{A}$ was set to $2.5 \times 10^{-29} \mathrm{~cm}^{6} \mathrm{~s}^{-1}$. The

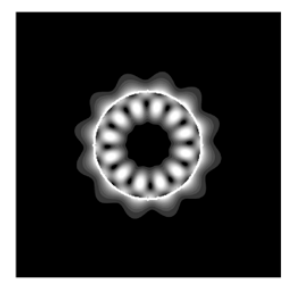

(a)

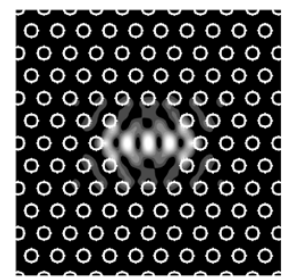

(c)

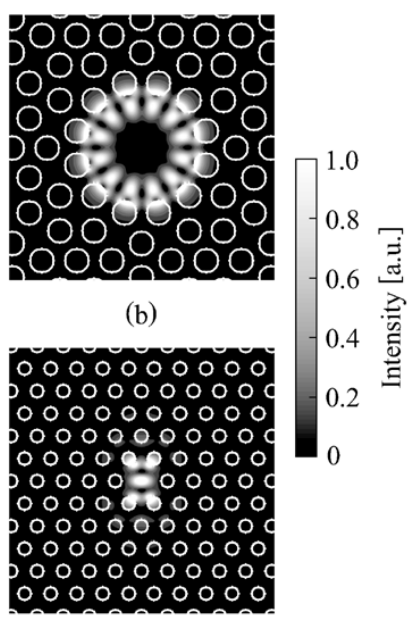

(d)
Fig. 1. Cavity models and electric field distributions $E^{2}\left(=E_{x}^{2}+E_{y}^{2}\right)$ of laser modes, which are calculated by FDTD method. (a) MDL. (b) QPC. (c) PC-H2. (d) PC-H1.

spontaneous emission rate is given by $\tau_{\mathrm{sp}}^{-1}=B N$, where $B$ was set to $2.0 \times 10^{-10} \mathrm{~cm}^{3} \mathrm{~s}^{-1}$. The photon lifetime $\tau_{\mathrm{ph}}$ was fixed to $2.23 \mathrm{ps}$, which corresponds to a cavity $Q$ factor of 3000 at a wavelength of $1.55 \mu \mathrm{m}$ and additional internal loss $\alpha_{i}=5 \mathrm{~cm}^{-1}$. The average threshold carrier density $\bar{N}_{\text {th }}$ is approximated as

$$
\bar{N}_{\mathrm{th}}=N_{0} \exp \left(\frac{n_{g}}{c \Gamma_{z} G_{0} \tau_{p h}}\right) .
$$

Substituting above values into (5), $\bar{N}_{\text {th }}$ is calculated to be $2.4 \times$ $10^{18} \mathrm{~cm}^{-3}$. In the first part of the calculation, the spontaneous emission factor $C$ and the diffusion constant $D$ were fixed to be 0.1 and $2 \mathrm{~cm}^{2} \mathrm{~s}^{-1}$, respectively, as standard values of GaInAsP microlasers [4], [12], [21], [23]. For the estimation of threshold power $p_{\text {th }}=P_{\mathrm{th}}\left(\pi R_{e x}^{2}\right) d$, the total thickness $d$ of CS-QWs was set to $20 \mathrm{~nm}$. The cell size $\Delta x$ and $\Delta y$ were $15 \mathrm{~nm}$ in both the FDTD and rate equation analyses. $\Delta t$ in the FDTD analysis was as short as 0.025 fs due to the fast oscillation of lightwaves, while it was 100 fs for the slower behavior of carriers and photons in rate equations.

\section{CARRIER DENSITY Distributions AND LASING CHARACTERISTICS}

As examples of microlasers, we modeled a MDL of $1.77 \mu \mathrm{m}$ diameter, 12-fold symmetric QPC [20] (the lattice constant $a=$ $585 \mathrm{~nm}$ and the hole diameter $2 r=420 \mathrm{~nm}$ ) with the sevenhole-missing defect, triangular lattice PC ( $a=435 \mathrm{~nm}$ and $2 r=240 \mathrm{~nm}$ ) with the seven-hole-missing defect (H2 defect) and PC with the single defect (H1 defect), as shown in Fig. 1. In the FDTD analysis, whispering gallery modes (WGMs) in the MDL (resonant wavelength $\lambda=1.575 \mu \mathrm{m}$ ) and in the QPC $(1.753 \mu \mathrm{m})$, a Fabry-Perot like mode in the PC-H2 $(1.663 \mu \mathrm{m})$, and a dipole mode in the PC-H1 $(1.489 \mu \mathrm{m})$ were obtained by the selective excitation of these wavelengths. Although other various modes are formed in the QPC and the PC-H2, we chose them as examples here. 

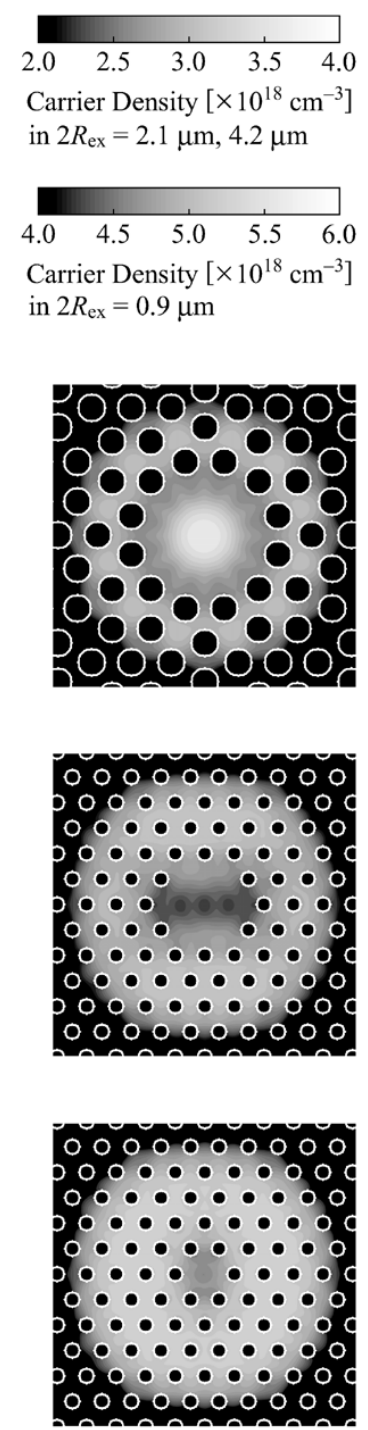
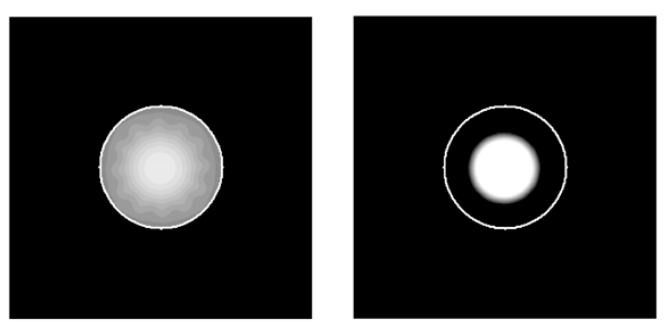

(a)
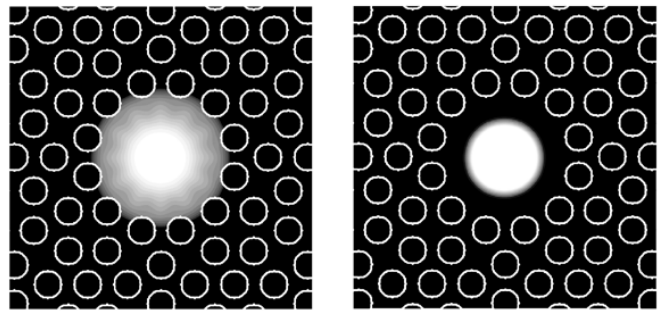

(b)

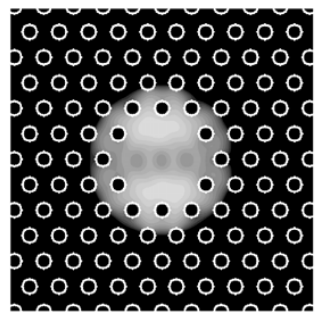

(c)

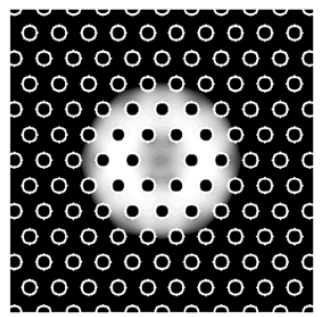

(d)
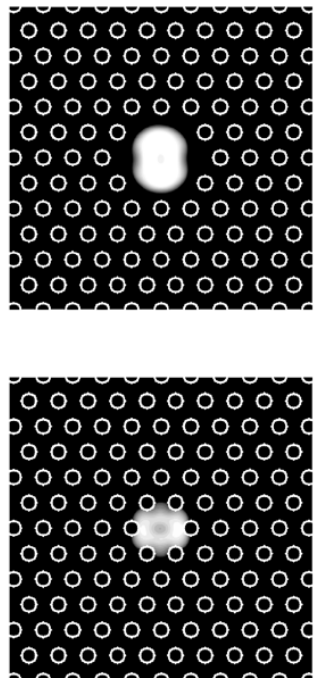

Fig. 2. Carrier distributions above threshold $\left(p \sim 2 p_{\text {th }}\right)$. (a) MDL. (b) QPC. (c) PC-H2. (d) PC-H1. From right to left, $2 R_{e x}=0.9 \mu \mathrm{m}, 2.1 \mu \mathrm{m}$, and $4.2 \mu \mathrm{m}$.

Fig. 2 shows carrier density distributions above threshold for $2 R_{e x}=0.9 \mu \mathrm{m}, 2.1 \mu \mathrm{m}$, and $4.2 \mu \mathrm{m}$. When the whole area of the MDL is uniformly pumped, the spatial hole burning occurs at the disk edge where the WGM is localized, and many carriers remain at the center, as shown in Fig. 2(a). When $2 R_{e x}$ is reduced to $0.9 \mu \mathrm{m}$ (similar situation to a current injection device with center posts), the hole burning becomes clearer. On this condition, direct pumping of the laser mode decreases, and the compensation of the hole burning by the carrier diffusion is not sufficient. In addition, the Auger recombination is enhanced at the center due to the high carrier density. In the QPC, the behavior of carriers is similar to that in the MDL, as shown in Fig. 2(b). The carrier loss by the surface recombination at holes is not so serious, compared with the hole burning. In other words, the carrier leakage to outside of the defect is blocked by the hole burning. In the PCs, the hole burning mainly occurs at the cavity center where their modes are localized, as shown in Figs. 2(c) and (d). Therefore, larger carrier leakage than in the QPC is expected. The detail of this matter will be discussed in Section V.
Fig. 3(a) shows log-log plots of lasing characteristics for $2 R_{e x}=2.1 \mu \mathrm{m}$. The unclear kink at threshold is due to the large $C$ factor assumed here. In the PCs and the QPC, the carrier leakage reduces the photon density of spontaneous emission below threshold. Fig. 3(b) shows the threshold power $p_{\text {th }}$ versus $2 R_{e x}$ characteristics. Each microlaser has its optimum pump diameter for threshold reduction. In the MDL, the optimum diameter is comparable to the disk diameter, because the WGM is directly excited on this condition. A low $p_{\text {th }}$ of $30 \mu \mathrm{W}$ is calculated for the uniformly pumped $1.77-\mu \mathrm{m}$-diameter MDL. This value roughly agrees to a measured value of $24 \mu \mathrm{W}$ for the 1.7- $\mu \mathrm{m}$-diameter MDL [4]. In the QPC and the PCs, $2 R_{e x}$ larger than the modal diameter increases the useless excitation and threshold, but a too small $2 R_{e x}$ also increases the threshold due to the strong hole burning and Auger recombination. As a result, optimum diameters are the same as or slightly larger than effective cavity diameters. This condition minimizes the carrier leakage, while keeping the direct pumping of the mode. For the QPC, PC-H2, and PC-H1, minimum threshold values of 48, 52 , and $42 \mu \mathrm{W}$ are calculated with optimum $2 R_{e x} \sim 2.0,1.5$, 


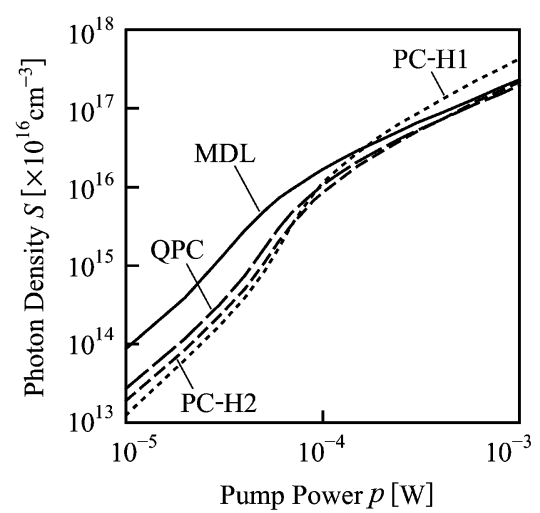

(a)

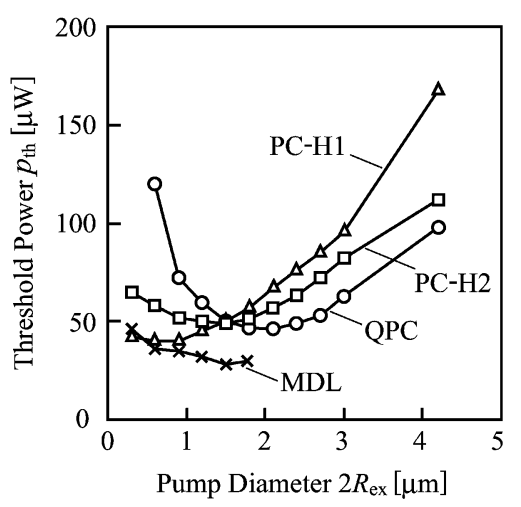

(b)

Fig. 3. Lasing characteristics for (a) $2 R_{e x}=2.1 \mu \mathrm{m}$ and (b) $p_{\text {th }}$ versus $2 R_{e x}$ characteristics. Cross, circle, square, and triangle denote MDL, QPC, PC-H2, and PC-H1, respectively.

and $0.8 \mu \mathrm{m}$, respectively. Thus far, a threshold irradiated power of $0.2 \mathrm{~mW}$ was reported for PC-H1 with $2 R_{e x}=3 \mu \mathrm{m}$ [13]. This value is converted to an effective threshold of $70 \mu \mathrm{W}$ by considering a reasonable pump efficiency of 0.33 [7]. This value also roughly agrees to the calculated value of $90 \mu \mathrm{W}$ for $2 R_{e x}=3 \mu \mathrm{m}$, as shown in Fig. 3(b). The small difference should be caused by the different structural parameters $(Q$ factor, material gain, etc.) and different experimental condition (intensity profile of pump light, etc.). The higher threshold values for these lasers than that for the MDL are due to the carrier leakage. However, the QPC maintains the low threshold for larger $2 R_{e x}$. This means that the threshold density $P_{\text {th }}$ in the QPC is lower than those of the PCs. It can be explained by the blocking effect of the carrier leakage. However, $p_{\text {th }}$ in the QPC is still higher that of the MDL. One essential factor is the $Q$ factor, which is fixed for all lasers in this calculation. Previously, we observed in three-dimensional FDTD calculation that the QPC cavity ideally has over seven times higher $Q$ than that of the MDL [7]. As will be shown in Section IV, such a high $Q$ allows a $p_{\text {th }}$ lower than that of the MDL.

\section{StRuctural DePENDENCE}

In this section, we discuss the dependence of the threshold $p_{\text {th }}$ on the surface recombination velocity $v_{s}$, diffusion constant $D$, and cavity $Q$. As a sample structure, we select QPC here. As shown in Fig. 4, smaller $v_{s}$ reduces $p_{\text {th }}$, but this reduction is saturated due to the carrier leakage. The typical $v_{s}$ for the GaInAsP system is $1.2 \times 10^{4} \mathrm{~cm} / \mathrm{s}$, and it can be reduced to $0.7 \times 10^{4} \mathrm{~cm} / \mathrm{s}$ by the $\mathrm{CH}_{4}$ plasma irradiation [24]. Even if $v_{s}$ is further reduced, $p_{\text {th }}$ is not improved so significantly. Fig. 5 shows the $p_{\text {th }}$ versus $D$ characteristics. The dependence absolutely changes for different $2 R_{e x}$. A smaller $D$ suppresses the carrier leakage and the surface recombination, while accelerating the hole burning. Normally, the former factor is dominant. However, when $2 R_{e x}$ is too small, the hole burning becomes serious and $p_{\text {th }}$ increases for smaller $D$. Fig. 6 shows the $p_{\text {th }}$ versus $Q$ factor characteristics. For any $2 R_{e x}, p_{\text {th }}$ decreases with $Q$, but the decrease is saturated when $Q$ is higher than 6000. It is influenced by the internal loss $\alpha_{i}$ assumed. The experimental $Q$ factor of the MDLs is typically 3000. If $Q \sim 10000$ and $2 R_{e x}=2.1 \mu \mathrm{m}$ (nearly equal to the defect diameter) are

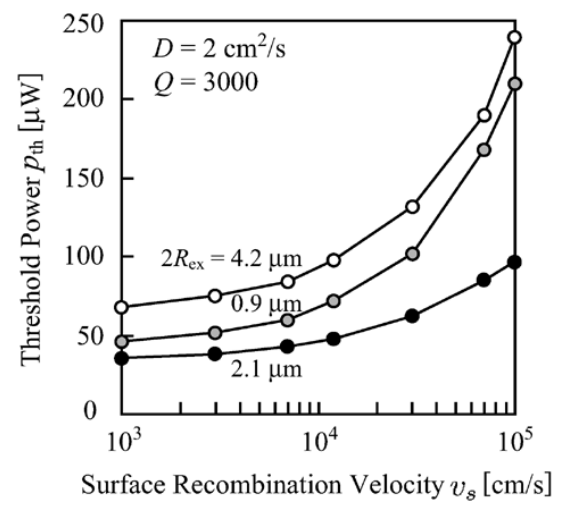

Fig. 4. $p_{\text {th }}$ versus $v_{s}$ characteristics for QPC.

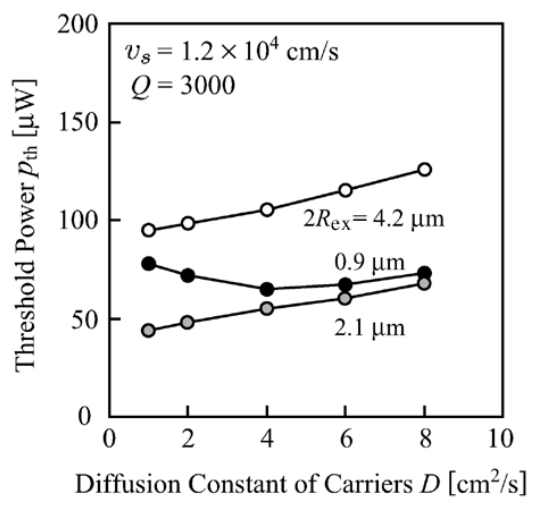

Fig. 5. $p_{\text {th }}$ versus $D$ characteristics for QPC.

realized for the QPC, $p_{\mathrm{th}}$ will be lower than that of the MDL. As mentioned in Section III, we can practically expect such a high $Q$ and low threshold.

\section{EFFective SuRface ReCombination Velocity}

The threshold density $P_{\text {th }}$ is approximately related with the average threshold carrier density $\bar{N}_{\text {th }}$ as

$$
\eta \frac{P_{\mathrm{th}}}{\hbar \omega}=B \bar{N}_{\mathrm{th}}^{2}
$$




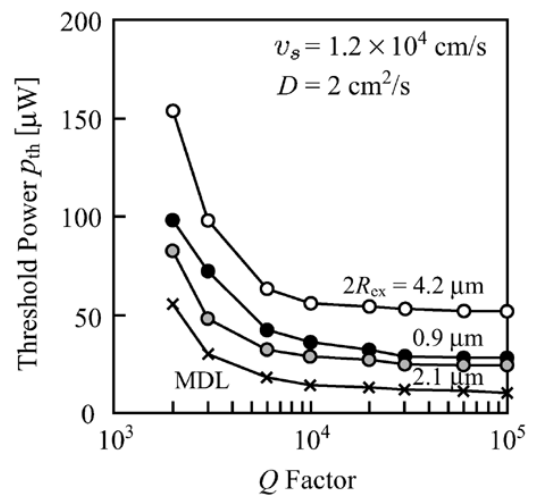

Fig. 6. $\quad p_{\text {th }}$ versus $Q$ factor characteristics for QPC. Cross plots are for MDL.

TABLE I

Summary of EFFeCtive CAVITy DiAmETER, THRESHOLD, EFFICIENCY, AND EFFECTIVE SURFACE RECOMBINATION VELOCITY

\begin{tabular}{c|c|c|c|c}
\hline & MDL & QPC & PC-H2 & PC-H1 \\
\hline \hline $2 R_{\text {eff }}[\mu \mathrm{m}]$ & 1.77 & 1.83 & 1.26 & 0.63 \\
\hline$p_{\text {th1 }}[\mu \mathrm{W}]$ & 23 & 27 & 19 & 12 \\
\hline$p_{\text {th2 }}[\mu \mathrm{W}]$ & 30 & 48 & 52 & 42 \\
\hline$\eta[\%]$ & 48 & 36 & 25 & 21 \\
\hline$\xi[\%]$ & 29 & 20 & 18 & 20 \\
\hline$\zeta[\%]$ & 23 & 44 & 57 & 59 \\
\hline$v_{\text {eff }}[\mathrm{cm} / \mathrm{s}]$ & $1.2 \times 10^{4}$ & $3.2 \times 10^{4}$ & $5.1 \times 10^{4}$ & $5.3 \times 10^{4}$ \\
\hline & & & &
\end{tabular}

where $\eta$ is the internal quantum efficiency, which is defined as the ratio of carriers effectively contributing to the lasing. In the QPC and the PCs, $\eta$ is reduced by the radiative recombination not coupled to the laser mode, Auger recombination, carrier leakage to outside of the defect, and surface recombination. Let us define $\xi$ and $\zeta$ as ratios of carriers for the former two factors and for the latter two factors, respectively. They satisfy the relation

$$
\eta+\xi+\zeta=1 .
$$

For the QPC and the PCs, we first consider here the effective circular cavities like the MDL, which internally touch with the innermost holes. The diameter $2 R_{\text {eff }}$ of the effective cavity is $1.83 \mu \mathrm{m}$ for the QPC, $1.26 \mu \mathrm{m}$ for the PC-H2, and $0.63 \mu \mathrm{m}$ for the PC-H1. We first assume that effective cavities are surrounded by air with no surface recombination at the boundary $\left(v_{s}=0 \mathrm{~cm} / \mathrm{s}\right)$. Then, the following relation is obtained:

$$
\left(1-\xi^{\prime}\right) \frac{P_{\mathrm{th} 1}}{\hbar \omega}=B \bar{N}_{\mathrm{th}}^{2}
$$

where the prime indicates $\xi$ without carrier leakage and surface recombination. The threshold density $P_{\mathrm{th} 1}$ in this situation is calculated by the rate equation analysis assuming the uniform pumping $\left(2 R_{e x}=2 R_{\text {eff }}\right)$. As shown in Table I, the corresponding $p_{\text {th } 1}$ is roughly proportional to the cavity area $\pi R_{\text {eff }}^{2}$.

Next, we assume full cavity models including outside of the defect and also assume an actual surface recombination velocity of $v_{s}=1.2 \times 10^{4} \mathrm{~cm} / \mathrm{s}$. Then, $P_{\mathrm{th} 2}$ and corresponding $p_{\mathrm{th} 2}$ are calculated. As shown in Table I, $p_{\text {th2 }}$ is considerably higher than $p_{\text {th1 }}$. To explain this, we define the effective surface recombination velocity $v_{\text {eff }}$, which includes the total effect by the surface recombination and the carrier leakage. Then

$$
\frac{P_{\mathrm{th} 2}}{\hbar \omega}=\frac{P_{\mathrm{th} 1}}{\hbar \omega}+v_{\mathrm{eff}} \frac{2 \pi R_{\mathrm{eff}}}{\pi R_{\mathrm{eff}}^{2}} \bar{N}_{\mathrm{th}}=\frac{B \bar{N}_{\mathrm{th}}^{2}}{1-\xi^{\prime}}+\frac{2 v_{\mathrm{eff}} \bar{N}_{\mathrm{th}}}{R_{\mathrm{eff}}} .
$$

The second term of (9) shows the effective surface recombination. Since $\zeta$ denotes the ratio of carrier loss by the effective surface recombination, the following relation is obtained:

$$
\zeta \frac{P_{\mathrm{th} 2}}{\hbar \omega}=\frac{2 v_{\mathrm{eff}} \bar{N}_{\mathrm{th}}}{R_{\mathrm{eff}}} .
$$

From (9) and (10)

$$
\left(1-\xi^{\prime}\right)(1-\zeta) \frac{P_{\mathrm{th} 2}}{\hbar \omega}=B \bar{N}_{\mathrm{th}}^{2} .
$$

By comparing (6) and (11), we obtain

$$
\eta=\left(1-\xi^{\prime}\right)(1-\zeta)=1-\xi^{\prime}(1-\zeta)-\zeta .
$$

Thus, from (7) and (12)

$$
\xi=\xi^{\prime}(1-\zeta)
$$

Table I summarizes $\eta, \xi, \zeta$, and $v_{\text {eff }}$ calculated by the abovementioned procedure. In the MDL, $\eta$ is higher than in other cavities due to the low $\zeta$. The calculated $v_{\text {eff }}$ is almost the same as the assumed $v_{s}$. This means that approximations of (5) and (6) are well consistent with the rate equation analysis. On the other hand, in the QPC, PC-H2, and PC-H1, $\eta$ is 0.75 times, 0.52 times, and 0.44 times smaller than in the MDL, respectively, due to the carrier leakage. The relatively high value for the QPC is attributed to the low $\zeta$ arising from the above-mentioned blocking of the carrier leakage. In general, the decrease in $R_{\text {eff }}$ increases $v_{\text {eff }}$. Therefore, it increases $\zeta$ more rapidly by the relation of (10). There are no significant differences of $\zeta$ between $\mathrm{PC}-\mathrm{H} 2$ and $\mathrm{PC}-\mathrm{H} 1$. This may be caused by assumed modal profiles for these cavities, both of which are localized at the defect center. As a result, $v_{\text {eff }}$ is 2.7 times, 4.2 times, and 4.4 times larger than $v_{s}$ in the QPC, $\mathrm{PC}-\mathrm{H} 2$, and $\mathrm{PC}-\mathrm{H} 1$, respectively. The relatively higher threshold $p_{\mathrm{th} 2}$ in the QPC is simply arising from the larger defect size. If the defect size of the QPC is reduced by adjusting the lattice and hole size, $p_{\mathrm{th} 2}$ will be lower than those of PCs. Fig. 7 shows the relation between $v_{\text {eff }}, v_{s}$ and $p_{\text {th2 }}$ in the MDL and the QPC. In the MDL, $v_{\text {eff }}$ always equals to $v_{s}$. On the other hand, in the QPC, a large difference is observed between $v_{\text {eff }}$ and $v_{s}$. When $v_{s}$ is very large, most of carriers are lost only at innermost holes around the defect by the surface recombination. Therefore, the carrier leakage cannot be a dominant factor, and the QPC and the PCs behave like MDLs. When $v_{s}$ is sufficiently small, the surface recombination at innermost holes decreases, and the carrier leakage expands the difference between $v_{\text {eff }}$ and $v_{s}$. 


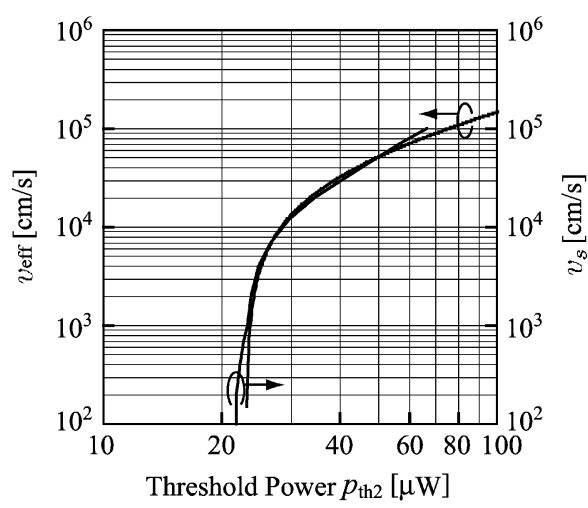

(a)

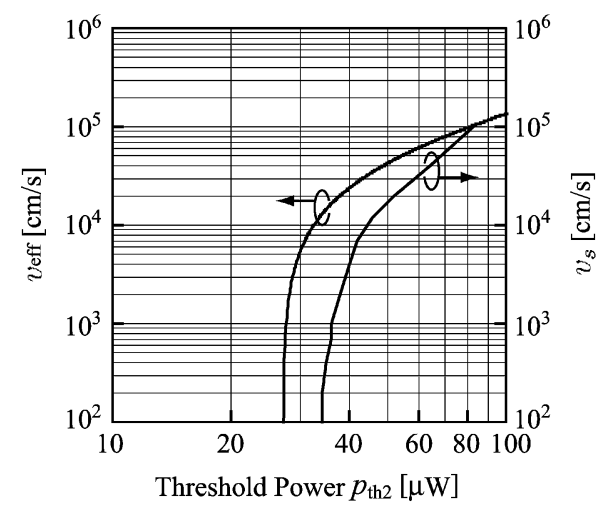

(b)

Fig. 7. Relation among $v_{\text {eff }}, v_{s}$, and $p_{\text {th2 }}$. (a) MDL. (b) QPC.

\section{CONCLUSION}

We performed the 2-D laser rate equation analysis, which was combined with the FDTD mode analysis, and observed the behavior of carriers and photons in photonic microlasers. Since the surface recombination and the carrier diffusion are precisely taken into account, distribution of the gain and absorption and the special hole burning can be quantitatively evaluated. Moreover, these results are obtained in time domain. Therefore, this method is applicable to the dynamic calculation of laser characteristics [14]. In the calculated results, the MDL having no carrier leakage to the outer space showed the lowest threshold. Compared with PCs, the QPC showed a relatively low threshold density and a high efficiency due to the blocking of the carrier leakage by the WGM. To achieve a lower threshold and higher efficiency in PCs and the QPC, suppression of the carrier leakage by separating the defect area from the PC or QPC area will be an important issue.

\section{REFERENCES}

[1] H. Yokoyama and K. Ujihara, Eds., Spontaneous Emission and Laser Oscillation in Microcavities. Boca Raton, FL: CRC, 1995.

[2] T. Baba, "Photonic crystals and microdisk cavities based on GaInAsP-InP system," IEEE J. Sel. Top. Quantum. Electron., vol. 3, pp. 808-830, May 1997.

[3] M. Pelton, J. Vuèkoviæ, G. S. Solomon, A. Scherer, and Y. Yamamoto, "Three-dimensionally confined modes in micropost microcavities: Quality factors and Purcell factors," IEEE J. Quantum Electron., vol. 38, no. 2, pp. 170-177, Feb. 2002.

[4] T. Baba and D. Sano, "Low threshold lasing and Purcell effect in microdisk lasers at room temperature," IEEE J. Sel. Top. Quantum Electron., vol. 9, no. 5, pp. 1340-1346, Sep.-Oct. 2003.

[5] S. L. McCall, A. F. J. Levi, R. E. Slusher, S. J. Pearton, and R. A. Logan, "Whispering-gallery mode microdisk lasers," Appl. Phys. Lett., vol. 60, pp. 289-291, 1992.

[6] M. Fujita, R. Ushigome, and T. Baba, "Continuous wave lasing in GaInAsP microdisk injection laser with threshold current of $40 \mu \mathrm{A}$," Electron. Lett., vol. 36, no. 9, pp. 790-791, Apr. 2000.

[7] K. Nozaki, A. Nakagawa, D. Sano, and T. Baba, "Ultralow threshold and single-mode lasing in microgear lasers and its fusion with quasi-periodic photonic crystals," IEEE J. Sel. Top. Quantum Electron, vol. 9, no. 5, pp. 1355-1360, Nov.-Dec. 2003.

[8] J. L. Jewell, J. P. Harbison, A. Scherer, Y. H. Lee, and L. T. Florez, "Vertical-cavity surface emitting lasers: Design, growth, fabrication, characterization," IEEE J. Quantum Electron., vol. 27, no. 6, pp. 1332-1347, Jun. 1991.

[9] T. Baba, T. Hamano, F. Koyama, and K. Iga, "Spontaneous emission factor of microcavity DBR surface-emitting laser," IEEE J. Quantum Electron., vol. 27, pp. 1347-1358, 1991.

[10] O. Painter, R. K. Lee, A. Scherer, A. Yariv, J. D. O'Brien, D. D. Dapkus, and I. Kim, "Two dimensional photonic bandgap defect mode laser," Science, vol. 284, pp. 1819-1821, 1999.
[11] K. Inoshita and T. Baba, "Lasing at bend, branch, and intersection of photonic crystal waveguides," Electron. Lett., vol. 39, pp. 844-845, May 2003.

[12] H. Y. Ryu, M. Notomi, E. Kuramochi, and T. Segawa, "Large spontaneous emission factor $(>0.1)$ in the photonic crystal monopole-mode laser," Appl. Phys. Lett., vol. 84, pp. 1067-1069, 2004.

[13] M. Loncarr, T. Yoshie, A. Scherer, P. Gogna, and Y. Qiu, "Low-threshold photonic crystal laser," Appl. Phys. Lett., vol. 81, pp. 2680-2682, 2002.

[14] T. Baba, D. Sano, K. Nozaki, K. Inoshita, Y. Kuroki, and F. Koyama, "Observation of fast spontaneous emission decay in GaInAsP photonic crystal point defect nanocavity at room temperature," Appl. Phys. Lett., vol. 85, pp. 3989-3991, 2004.

[15] T. Yoshie, A. Scherer, H. Chen, D. Huffaker, and D. Deppe, "Optical characterization of two-dimensional photonic crystal cavities with indium arsenide quantum dot emitters," Appl. Phys. Lett., vol. 79, pp. 114-116, 2001.

[16] J. Vuèkoviæ and Y. Yamamoto, "Photonic crystal microcavities for cavity quantum electrodynamics with a single quantum dot," Appl. Phys. Lett., vol. 82, pp. 2374-2376, 2003.

[17] K. Hennessy, C. Reese, A. Badolato, C. F. Wang, A. Imamoğlu, P. M. Petroff, E. Hu, G. Jin, S. Shi, and D. W. Prather, "Square-lattice photonic crystal microcavities for coupling to single InAs quantum dots," Appl. Phys. Lett., vol. 83, pp. 3650-3652, 2003.

[18] M. E. Zoorob, M. D. B. Charlton, G. J. Parker, J. J. Baumberg, and M. C. Netti, "Complete photonic bandgaps in 12-fold symmetric quasi-crystals," Nature, vol. 404, pp. 740-743, 2000.

[19] M. Notomi, H. Suzuki, T. Tamamura, and K. Edogawa, "Lasing action due to the two-dimensional quasi-periodicity of photonic quasi-crystals with a Penrose lattice," Phys. Rev. Lett., vol. 92, p. 123906, 2004.

[20] K. Nozaki and T. Baba, "Quasiperiodic photonic crystal microcavity laser," Appl. Phys. Lett., vol. 84, p. 4875, 2004.

[21] M. Fujita, A. Sakai, and T. Baba, "Ultrasmall and ultralow threshold GaInAsP-InP microdisk injection lasers: Design, fabrication, lasing characteristic, and spontaneous emission factor," IEEE J. Sel. Top. Quantum Electron., vol. 5, no. 3, pp. 673-681, May-Jun. 1999.

[22] Y. Suematsu, Ed., Semiconductor Lasers and Integrated Optics. Tokyo, Japan: Ohmsha, 1984. (in Japanese).

[23] H. G. Park, J. K. Hwang, J. Huh, H. Y. Ryu, S. H. Kim, J. S. Kim, and Y. H. Lee, "Characteristics of modified single-defect two-dimensional photonic crystal lasers," IEEE J. Quantum Electron., vol. 38, pp. 1353-1365, 2002.

[24] H. Ichikawa, K. Inoshita, and T. Baba, "Reduction in surface recombination of GaInAsP/InP microcolumns by $\mathrm{CH}_{4}$ plasma irradiation," Appl. Phys. Lett., vol. 78, pp. 2119-2121, 2001.

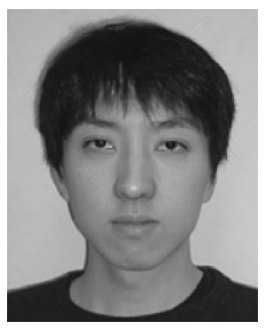

Kengo Nozaki was born in Niigata Prefecture, Japan, on July 15, 1980. He received the B.E. and M.E. degrees from the Department of Electrical and Computer Engineering, Yokohama National University (YNU), Yokohama, Japan, in 2003 and 2005, respectively. $\mathrm{He}$ is currently working towards the Ph.D. degree in photonic crystal lasers at Yokohama National University.

Mr. Nozaki is a member of the Japan Society of Applied Physics (JSAP). 


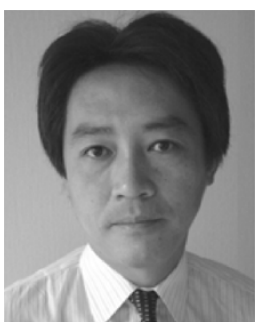

Toshihiko Baba (M'03) received the B.E., M.E., and Ph.D. degrees from Yokohama National University (YNU), Yokohama, Japan, in 1985, 1987, and 1990, respectively. During his Ph.D. work, he had been engaged in antiresonant reflecting optical waveguides (ARROWs).

In 1990, he joined the Tokyo Institute of Technology as a Research Associate, and reported the spontaneous emission control in vertical cavity surface emitting lasers (VCSELs) and the first room temperature $\mathrm{cw}$ operation of long wavelength VCSELs. In 1994, he became an Associate Professor at YNU, and started research on photonic crystals (PCs) and microdisk lasers (MDLs). Regarding PCs, he reported the first fabrication and characterization of an InP-based light emitters and a line-defect waveguide at lightwave frequencies. He also studied enhancement of light extraction efficiency and passivation techniques. In 2005, he became a Full Professor at YNU. His recent interests are PC-based nanocavity lasers, optical buffer, LEDs, VCSEL, and so on. Regarding MDLs, he achieved the room temperature $\mathrm{cw}$ operation with the record low threshold, reported the microgear and photonic molecule structures, and demonstrated a MDL-based near-field sensor. Besides these, he is also active on a deep grating distributed Bragg reflector for short cavity lasers and $\mathrm{Si}$ photonics based on nanowire waveguides.

Dr. Baba is a member of the Institute of Electrical, Information, and Communication Engineers (IEICE), IEEE/LEOS, the Japan Society of Applied Physics, and the American Physics Society. He received the Niwa Memorial Prize in 1991, the Best Paper Award at the Micro-Optic Conference in 1993 and 1999, respectively, the Paper Award and Academic Encouragement Award from the IEICE in 1994, and the Marubun Research Encouragement Award in 2000. 